\title{
AVALIAÇÃO DO RUÍDO OCUPACIONAL EM UM RESTAURANTE NUM MUNICÍPIO DO MATO GROSSO DO SUL
}

\author{
Maria Salete Vaceli Quintilio ${ }^{1}$, Patricia Arruda de Souza Alcarás ${ }^{1}$, Laís da Silva Martins ${ }^{2}$ \\ ${ }^{1}$ Docente e ${ }^{2}$ Discente do Curso de Fonoaudiologia da UNOESTE.
}

\begin{abstract}
RESUMO
Em ambientes ocupacionais, que podem gerar ruídos elevados, é recomendado o uso de equipamentos de proteção individuais (EPIs), além de medidas para diminuição do nível de ruídos, a fim de proteger a audição dos trabalhadores expostos. Em restaurantes e cozinhas industriais observa-se um nível de ruído bastante intenso, porém é escasso o número de locais onde há, efetivamente, a utilização de equipamentos para a proteção da audição dos funcionários. Este estudo teve por objetivo mensurar os níveis do ruído ambiental produzidos em um restaurante localizado num município do interior do Mato Grosso do Sul. Os níveis de ruído foram medidos durante seis dias, em dois horários, com um decibelímetro digital Minipa, em quatro pontos no interior do local e um no exterior. A partir das medidas coletadas e analisadas, observouse que o nível de ruído médio no salão do restaurante foi de 98,9 dB(A), na cozinha 103,9 dB(A) e no exterior de 105,4 dB(A). Pode-se observar, ainda, que no horário de almoço o nível de ruído foi maior que no horário do jantar, em todos os locais. Assim, pode-se concluir que o restaurante estudado é candidato à implementação de um Programa de Conservação Auditiva, visto haver elevados níveis de pressão sonora em todos os ambientes mensurados.
\end{abstract}

Palavras-chave: ruído ocupacional, audição, perda auditiva.

\section{EVALUATION OF THE OCCUPATIONAL NOISE IN A RESTAURANT IN A TOWN IN MATO GROSSO DO SUL STATE}

\begin{abstract}
In occupational settings that can generate high levels of noise, it is recommended the use of a personal protective equipment and other methods to decrease the noise level, in order to protect the hearing of workers exposed to such settings. In restaurants and industrial kitchens there is a quite intense noise level, however the number of establishments where in fact any kind of equipment to protect the employees' hearing has been used has been rare. This study intended to measure environmental noise levels in a restaurant located in a town in Mato Grosso do Sul, Brazil. Noise levels were measured through a period of six days, two times a day, using a digital decibelmeter Minipa, at four points inside the restaurant and another one outside. From the data collected and analyzed it was found that the average noise level at the restaurant's were $98.9 \mathrm{~dB}(\mathrm{~A})$ for the lounge, $103.9 \mathrm{~dB}(\mathrm{~A})$ for the kitchen and $105.4 \mathrm{~dB}(\mathrm{~A})$ for the outside. It can be observed that at lunch time the noise level was higher than at dinner time for all locations. The study concludes that the referred restaurant is a candidate for the implementation of a Hearing Conservation Program.
\end{abstract}

Keywords: occupational noise, hearing, hearing loss. 


\section{INTRODUÇÃO}

Diariamente, estamos expostos a ruídos de diversas intensidades. $O$ processo de industrialização alcançado através dos avanços da tecnologia e modernidade favoreceu 0 aumento de doenças ocupacionais, sendo o ruído considerado o agente mais nocivo presente nos ambientes insalubres (RIOS, 2007).

No Brasil, a Norma Regulamentadora NR 15 (BRASIL, 1978) permite uma exposição de até 8 horas diárias a ruídos de $85 \mathrm{~dB}$ em nível de pressão sonora (dBNPS). Conforme aumenta o nível de ruído, diminui o tempo de exposição permitido pela NR 15. A exposição por tempos maiores pode acarretar em perda auditiva, denominada Perda Auditiva Induzida por Ruído (PAIR). Em restaurantes e cozinhas industriais observa-se um nível de ruído bastante intenso, porém é escasso o número de locais onde há, efetivamente, a utilização de equipamentos para a proteção da audição dos funcionários.

Marques e Costa (2006) e Gonçalves et al. (2009) relataram que a exposição a elevados níveis de pressão sonora é um risco à saúde do trabalhador, pois além de causar alterações no sistema auditivo, provocam perturbações no ambiente de trabalho, na qualidade do sono, no descanso e nas situações dialógicas.

Mello e Waismann (2004) relataram que os efeitos do ruído na audição podem ser divididos em três categorias: mudança temporária do limiar auditivo (MTLA) ou Temporary Threshold Shift (TTS), trauma acústico e mudança permanente do limiar (MPLA) ou Permanent Threshold Shift (PTS); sendo esta última a Perda Auditiva Induzida por Ruído PAIR.

Nudelmann (2001) referiu que o indivíduo exposto a elevados níveis de pressão sonora pode apresentar: perda auditiva, zumbidos, dificuldades para compreender a fala e localizar a fonte sonora, algiacusia (dor a sons) e sensação de ouvido tampado.

Atti et al. (2000) salientaram que a PAIR ocupa o segundo lugar entre as doenças mais frequentes do aparelho auditivo, e esta doença, poderia não só afetar a audição, mas também o equilíbrio.

De acordo com Palma (1999), os efeitos do ruído são mais eminentes e característicos na audição, pois, ao atingi-la, o ruído comprometeria a compreensão da fala do individuo. Este autor, em seus estudos, comparou a compreensão de sentenças no silêncio e no ruído, entre os indivíduos com PAIR, cujo resultado ressaltou o comprometimento da compreensão de sentenças, tanto no silêncio quanto na presença de ruído competitivo. Assim, o resultado do estudo demonstrou que a PAIR implica em um processo de interação com o meio social afetado. Vieira citado por Palma (1999) referiu que o indivíduo portador de PAIR só será capaz de perceber a lesão auditiva quando sua comunicação já estiver prejudicada.

Muitos estudos mostram a presença de altos níveis de ruído nas cozinhas de restaurantes, entretanto, ressaltam a não utilização de equipamentos para a proteção auditiva nessa população. Com objetivo em verificar o nível de ruído ambiental dentro das cozinhas de cinco restaurantes localizados no município de Presidente Prudente - SP, Lopreto (2006) concluiu que o ruído presente no interior desses restaurantes é significativamente elevado (nível de pressão sonora variando entre 61 a 90 dBNPS), podendo este lesionar o sistema auditivo.

Outro fator agravante e significativo no sujeito exposto ao ruído é a presença do zumbido. O zumbido é o primeiro sinal de alerta de que a exposição está lesionando o sistema auditivo, podendo indicar maior susceptibilidade à 
lesão pelo ruído (OGIDO et al., 2009). A maioria das pessoas sente a presença do zumbido depois de ir a um conserto de música, e nestes casos, o zumbido costuma desaparecer em poucas horas (PFEIFFER et al., 2007). Algumas vezes o zumbido pode ser o sintoma de uma perda auditiva passageira, conhecida como Perda Auditiva Temporária (TTS - Tempory Threshold Sfhit) (SILVA, 1999).

De acordo com López Ugalde et al. (2000) um terço da população mundial apresenta perda auditiva causada por ruídos de alta intensidade e referem que cada vez mais podemos encontrar o ruído em todos os locais (casa, espaços de recreação, escolas etc), o que faz com que todos os membros da sociedade fiquem sujeitos à PAIR.

O Brasil é um dos países com maior incidência de locais com elevados níveis de ruído. Em grandes metrópoles, como São Paulo e Rio de Janeiro, o ruído pode alcançar picos de 105 $\mathrm{dB}(\mathrm{A})$. Segundo Bento et al. (1998), quando o indivíduo frequenta ambiente com nível sonoro superior a $85 \mathrm{~dB}$, durante uma jornada de trabalho, tem nesta situação um risco potencial para a ocorrência de lesão auditiva.

Para Becker et al. (1999), a derradeira solução para a PAIR ainda é a prevenção, que demanda educação do trabalhador para uso dos protetores auriculares, tratamento acústico das máquinas, limitação do tempo de exposição ao ruído e profilaxia médica contra a lesão à audição prescrita pelo pessoal responsável pela saúde ocupacional.

Assim, a medição do ruído ambiental possibilita um mapeamento das áreas de maior risco, permitindo uma avaliação do local em questão. Deve-se, ainda, observar alguns fatores, tais como: nível de exposição individual, tipo de ruído, tipo de exposição e características do local (FERNANDES, 1999).
Por esse motivo, o presente estudo teve por objetivo medir os níveis de ruído ocupacional gerados em vários ambientes de um restaurante localizado num município do interior do Mato Grosso do Sul. A partir da análise do nível de pressão sonora medida nos vários pontos do local, pode-se avaliar a nocividade do ruído e verificar a necessidade do Programa de Conservação Auditiva (PCA) e de Equipamentos de Proteção Individual (EPI) no restaurante.

\section{MATERIAIS E MÉTODOS}

Este estudo se desenvolveu num restaurante localizado no interior do estado do Mato Grosso do Sul. Os níveis de pressão sonora (NPS) foram medidos utilizando um Decibelímetro Digital da marca Minipa MSL-1325, devidamente calibrado conforme as normas regulamentadoras NR-15. As medições foram obtidas posicionando o instrumento na altura equivalente à orelha dos funcionários, como é de praxe em medições de salas e ambientes fechados. Utilizou-se a curva de ponderação $A$, para ruídos contínuos e flutuantes, e a escala de tempo fast.

Obteve-se o valor mínimo e o máximo em cada medição, realizada em cinco pontos diferentes do restaurante, sendo três laterais, um no interior da cozinha e um do lado de fora do estabelecimento, em momentos distintos do processo de funcionamento do restaurante. As medidas foram coletadas nos horários de maior movimento na cozinha e no restaurante, ou seja, durante o almoço e o jantar, com os diversos equipamentos em funcionamento. A coleta ocorreu durante seis finais de semanas, pois trata-se do período de maior fluxo de clientes.

\section{RESULTADOS E DISCUSSÃO}

As Figuras 1 e 2 mostram os valores médios para os ambientes estudados, onde o salão compreende os pontos $A, B$ e $C$, a cozinha 


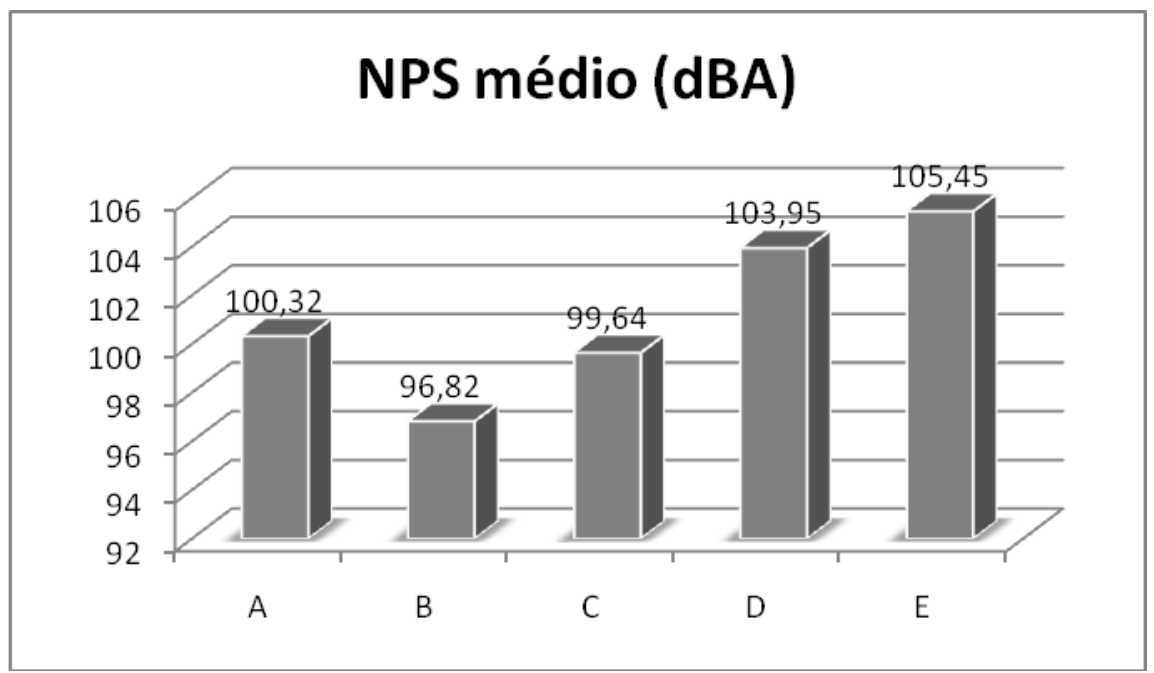

Figura 1. NPS médio para cada ponto no restaurante.

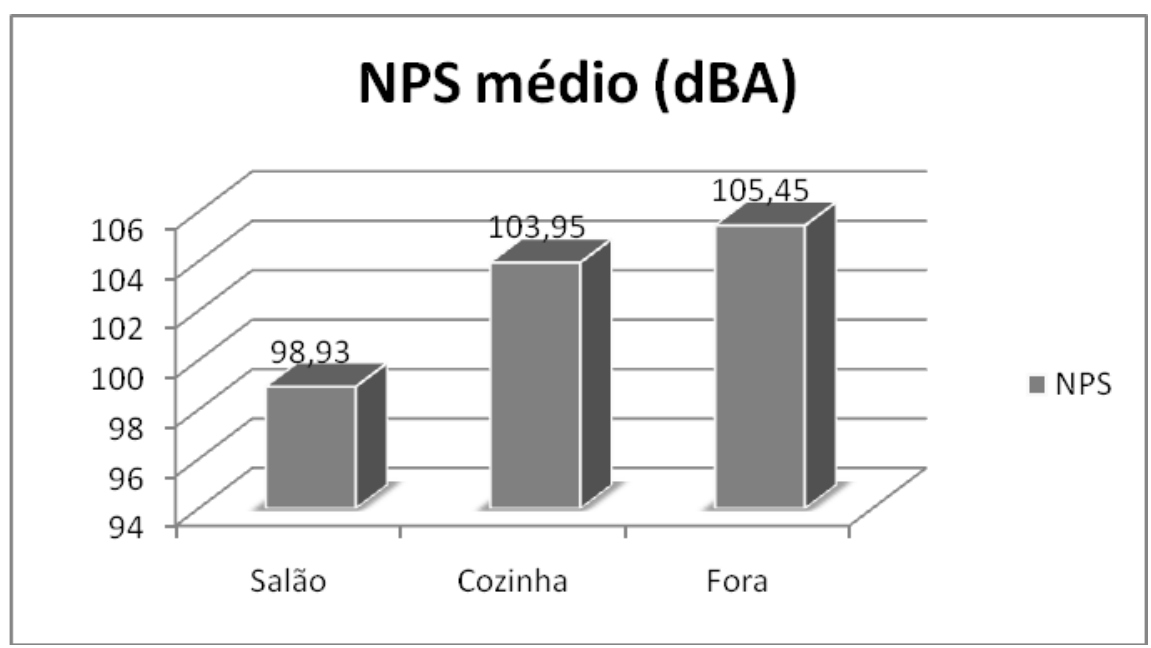

Figura 2. NPS médio por ambiente.

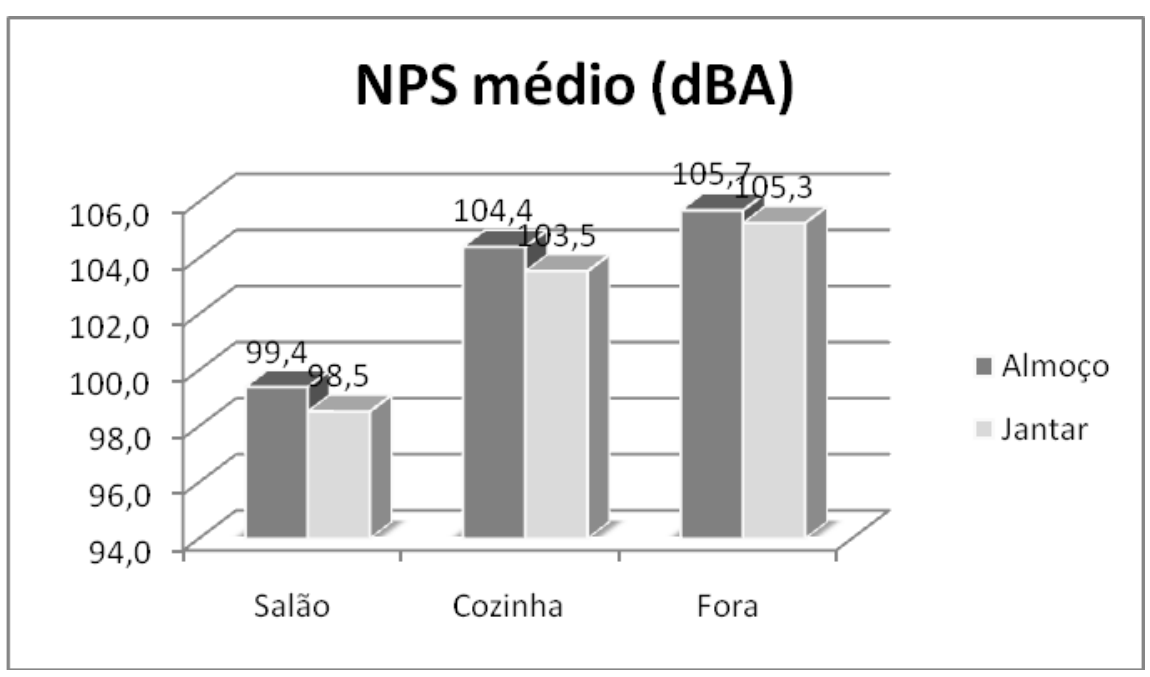

Figura 3. Medidas do NPS médio por ambiente e por horário. 
Fica claro que os valores alcançados superaram o limite de $85 \mathrm{~dB}$ por 8 horas, conforme recomendações estabelecidas pelas Normas Regulamentadoras - NR15 (BRASIL, 1978).

Ainda, observamos que, apesar dos profissionais estarem expostos a um ruído superior a 90 dBNPS, tanto no salão do restaurante quanto na cozinha, nenhum deles faz uso de proteção auditiva.

Como consequência, estudos realizados por Palma (1999) referiram que os níveis elevados de pressão sonora danificam as estruturas da cóclea, comprometendo a compreensão de fala do indivíduo, principalmente em ambientes com ruído competitivo. Além disso, outro sintoma pode ser apresentado pelo indivíduo exposto ao ruído, o zumbido.

Assim, no local analisado por essa pesquisa, pode-se concluir que os profissionais, independente do local em que atuam, estão expostos a elevados níveis de pressão sonora, podendo este ser desastroso para o sistema auditivo, como mostram as Figuras 1 e 2.

De acordo com a Figura 3, nota-se que o horário de ruído mais intenso foi durante 0 almoço, tanto no salão (NPS médio de 99,4 dB) quanto na cozinha (NPS médio de 104,4 dB).

Com relação ao nível de intensidade medido, notamos que os momentos de maior movimento no restaurante, o ruído na cozinha atingiu os $104 \mathrm{~dB}$, o que geralmente acontecia nos momentos em que várias pessoas conversavam ao mesmo tempo e existia mais de um equipamento ligado no local, tais como, liquidificadores, exaustores, etc.

Os resultados obtidos nesta pesquisa são corroborados pelos estudos realizados por Lopreto (2006), que mediu o ruído presente no interior das cozinhas de cinco restaurantes, onde observou que o nível máximo de pressão sonora foi de 90 dBNPS.
Entretanto, ao se comparar os valores no interior do local com os valores medidos na calçada do restaurante, o ruído no exterior foi significantemente mais intenso. Tal fato se justifica pelo intenso fluxo do tráfego de veículos, uma vez que o restaurante estudado localiza-se em uma avenida principal. Ao entrar no interior do salão do restaurante, foi observada imediata redução de ruído ambiental.

Os resultados das medições do nível sonoro ficaram acima dos valores recomendados pela NR15, pois a jornada de trabalho desses profissionais é muito extensa (maior que oito horas diárias). O presente estudo revelou a necessidade de redução dos níveis de ruído em vista das possíveis repercussões na saúde e na vida da equipe de profissionais. No entanto, como não foi averiguado se existe um rodízio de funções ou um intervalo de descanso auditivo, seria de interesse propor um estudo adicional utilizando-se um dosímetro a fim de medir a exposição diária individual de ruído.

De acordo com Amaral (2002) a perda auditiva ocasionada pelo ruído é irreversível e este é um dado preocupante, pois segundo Vieira citado por Palma (1999), o indivíduo portador de PAIR só seria capaz de perceber a lesão auditiva quando sua comunicação já estivesse prejudicada. Isto pode estar ocorrendo com os funcionários dos restaurantes, já que a PAIR é de instalação lenta e insidiosa.

\section{CONCLUSÃO}

Após a análise dos resultados, concluiuse que 0 nível de ruído produzido nas dependências do restaurante estudado são de forte intensidade, acima dos $85 \mathrm{~dB}(\mathrm{~A})$ recomendados para uma exposição diária de 8 horas, de acordo com a NR 15, colocando em risco a saúde auditiva dos trabalhadores.

Também é de grande importância a investigação dos limiares auditivos dos 
funcionários com o intuito de investigar queixas devido aos efeitos do ruído.

Os resultados encontrados justificam a proposta de implantação de um Programa de Conservação Auditiva no restaurante, visando à diminuição dos níveis de ruído produzidos neste ambiente de trabalho.

\section{REFERÊNCIAS}

AMARAL M. et al. Estudo retrospectivo sobre a audição de trabalhadores expostos ao ruído. Revista CEFAC, p. 145-148, 2002.

ATTI, J. L. et al. Perda auditiva induzida por ruído. In: LOPES, A.C. et al. Caracterização dos limiares audiológicos em trabalhadores de urnas funerárias. Arquivos Internacionais de Otorrinolaringologia, vol.12, n.3, p.244-251, 2000.

BECKER, W.; NAUMANN, H. H.; PFALTZ, C. R. Otorrinolaringologia prática: diagnóstico e tratamento. 2. ed. São Paulo: Revinter, 1999.

BENTO, R.; MINITI, A.; MARONE, S. Tratado de otologia. 1. ed. São Paulo: Edusp, 1998.

BRASIL. Ministério do Trabalho e Emprego. Secretaria de Inspeção do Trabalho. Portaria № 3.214, 8 de junho de 1978. Disponível em <http://www.mte.gov.br/legislacao/portarias/1978/ p_19780608_3214.pdf > Acesso em: 13 de set de 2009 .

FERNANDES, J. Apostila sobre ruído e seus efeitos. Bauru: [s.n.], 1999.

LÓPEZ UGALDE, A. C. et al. Hipoacusia por ruído: um problema de salud y de conciencia pública. 2000. Disponível em: <http://bases.bireme.br/cgi-

bin/wxislind.exe/iah/online/>. Acesso em: 10 out. 2009.

LOPRETO, C. Cozinha de restaurantes: análise dos níveis de ruído. 2006. 46f. Monografia (Trabalho de Conclusão de Curso) - Faculdade de Fonoaudiologia, Universidade do Oeste Paulista, Presidente Prudente.

MARQUES, F. P.; COSTA, E. A. Exposição ao ruído ocupacional: alterações no exame de emissões otoacústicas. Revista Brasileira de Otorrinolaringologia, São Paulo, vol.72, n.3, 2006.

MELLO, A. P.; WAISMANN, W. Exposição ocupacional ao ruído e químicos industriais e seus efeitos no sistema auditivo: revisão de literatura. Arquivos Internacionais de Otorrinolaringologia, vol.8, p.226-34, 2004.

NUdelmanN, A. A. et al. PAIR - Perda Auditiva Induzida pelo Ruído. Rio de Janeiro: Revinter, 2001.

OGIDO, R.; COSTA, E. A.; MACHADO, H. C. Prevalência de sintomas auditivos e vestibulares em trabalhadores expostos a ruído ocupacional. Revista de Saúde Pública. São Paulo, vol. 43, n.2, 2009.

PALMA, D. C. Quando o ruído atinge a audição. Monografia (Especialização em Audiologia Clínica). 1999. Disponível em: http://www.cefac.br/library/teses/eec6828d182c8b 34af8bdc403d26c989.pdf. Acesso em: 02 jun. 2009.

PFEIFFER, M. et al. Intercorrência audiológica em músicos após um show de rock. Revista CEFAC, São Paulo, vol.9, n.3, 417-22, 2007.

RIOS, A. L. Implantação de um Programa de Conservação Auditiva: enfoque fonoaudiológico. 2007. 133f. Tese (Doutorado) - Programa de PósGraduação da Faculdade de Medicina de Ribeirão Preto da Universidade de São Paulo. Ribeirão Preto, SP.

SILVA, A. F. Mudança temporária do limiar auditivo. 1999. 65f. Monografia (Especialização) - Centro de Especialização em Fonoaudiologia CEFAC, Porto Alegre.

VIEIRA, S. I. Medicina básica do trabalho. In: PALMA, D.C. Quando o ruído atinge a audição. $1999 . \quad$ Disponível em: http://www.cefac.br/library/teses/eec6828d182c8b 34af8bdc403d26c989.pdf. Acesso em: 02 jun. 2009. 